\title{
Multiple Solutions of Semilinear Elliptic Prob- lems with Degenerate Boundary Conditions
}

\author{
Kazuaki Taira
}

Dedicated to the memory of Professor Kenji Asada (1946-2008)

\begin{abstract}
The purpose of this paper is to study a class of semilinear elliptic boundary value problems with degenerate boundary conditions which include as particular cases the Dirichlet and Robin problems. The approach here is distinguished by the extensive use of the ideas and techniques characteristic of the recent developments in the theory of partial differential equations. By making use of the Leray-Schauder degree, we prove very exact results on the number of solutions of our problem. The results here extend earlier theorems due to Berger-Podolak, CastroLazer and Ambrosetti-Mancini to the degenerate case.

Mathematics Subject Classification (2010). Primary 35J65; Secondary 35J20, 47H10.

Keywords. Semilinear elliptic boundary value problem, degenerate boundary condition, multiple solution, Leray-Schauder topological degree.
\end{abstract}

\section{Introduction and Main Results}

Let $\Omega$ be a bounded domain of Euclidean space $\mathbf{R}^{N}, N \geq 2$, with smooth boundary $\partial \Omega$; its closure $\bar{\Omega}=\Omega \cup \partial \Omega$ is an $N$-dimensional, compact smooth manifold with boundary. Let $A$ be a second-order, elliptic differential operator with real coefficients such that

$$
A u=-\sum_{i=1}^{N} \frac{\partial}{\partial x_{i}}\left(\sum_{j=1}^{N} a^{i j}(x) \frac{\partial u}{\partial x_{j}}\right)+c(x) u .
$$

Here:

(1) $a^{i j} \in C^{\infty}(\bar{\Omega})$ and $a^{i j}(x)=a^{j i}(x)$ for all $x \in \bar{\Omega}$ and $1 \leq i, j \leq N$, and there exists a positive constant $a_{0}$ such that

$$
\sum_{i, j=1}^{N} a^{i j}(x) \xi_{i} \xi_{j} \geq a_{0}|\xi|^{2} \quad \text { for all }(x, \xi) \in \bar{\Omega} \times \mathbf{R}^{N} \text {. }
$$


(2) $c \in C^{\infty}(\bar{\Omega})$ and $c(x) \geq 0$ in $\Omega$.

Let $B$ be a first-order, boundary condition with real coefficients such that

$$
B u=a\left(x^{\prime}\right) \frac{\partial u}{\partial \boldsymbol{\nu}}+b\left(x^{\prime}\right) u
$$

Here:

(3) $a \in C^{\infty}(\partial \Omega)$ and $a\left(x^{\prime}\right) \geq 0$ on $\partial \Omega$.

(4) $b \in C^{\infty}(\partial \Omega)$ and $b\left(x^{\prime}\right) \geq 0$ on $\partial \Omega$.

(5) $\partial / \partial \boldsymbol{\nu}$ is the conormal derivative associated with the operator $A$ :

$$
\frac{\partial}{\partial \boldsymbol{\nu}}=\sum_{i, j=1}^{N} a^{i j}\left(x^{\prime}\right) n_{j} \frac{\partial}{\partial x_{i}},
$$

where $\mathbf{n}=\left(n_{1}, n_{2}, \ldots, n_{N}\right)$ is the unit exterior normal to the boundary $\partial \Omega$.

Our fundamental hypotheses on the boundary condition $B$ are the following:

(H.1) $a\left(x^{\prime}\right)+b\left(x^{\prime}\right)>0$ on $\partial \Omega$.

(H.2) $b\left(x^{\prime}\right) \not \equiv 0$ on $\partial \Omega$.

It should be noticed that if $a\left(x^{\prime}\right) \equiv 0$ and $b\left(x^{\prime}\right) \equiv 1$ on $\partial \Omega$ (resp. $a\left(x^{\prime}\right) \equiv 1$ on $\partial \Omega$ ), then the boundary condition $B$ is the Dirichlet condition (resp. Robin condition). Moreover, it is easy to see that the boundary condition $B$ is nondegenerate (or coercive) if and only if either $a\left(x^{\prime}\right)>0$ on $\partial \Omega$ or $a\left(x^{\prime}\right) \equiv 0$ and $b\left(x^{\prime}\right)>0$ on $\partial \Omega$. Therefore, our boundary condition $B$ is a degenerate boundary value problem from an analytical point of view (cf. [13]). Amann [2] studied the boundary value condition (1.2) in the non-degenerate case where the boundary $\partial \Omega$ is the disjoint union of the two closed subsets $M$ and $\partial \Omega \backslash M$, each of which is an $(N-1)$ dimensional compact smooth manifold.

The intuitive meaning of condition (H.1) is that the absorption phenomenon occurs at each point of the set $M=\left\{x^{\prime} \in \partial \Omega: a\left(x^{\prime}\right)=0\right\}$, while the reflection phenomenon occurs at each point of the set $\partial \Omega \backslash M=\left\{x^{\prime} \in\right.$ $\left.\partial \Omega: a\left(x^{\prime}\right)>0\right\}$ (see [18]). On the other hand, condition (H.2) implies that the boundary condition $B$ is not equal to the purely Neumann condition.

In this paper we study the following semilinear homogeneous elliptic boundary value problem: Given a function $p(\xi)$ defined on $\mathbf{R}$, find a function $u(x)$ in $\Omega$ such that

$$
\begin{cases}A u-p(u)=0 & \text { in } \Omega, \\ B u=a\left(x^{\prime}\right) \frac{\partial u}{\partial \nu}+b\left(x^{\prime}\right) u=0 & \text { on } \partial \Omega .\end{cases}
$$

In order to study the homogeneous problem (1.3) in the framework of Hölder spaces, we consider the linear elliptic boundary value problem

$$
\begin{cases}A u=f & \text { in } \Omega, \\ B u=0 & \text { on } \partial \Omega\end{cases}
$$


in the framework of the Hilbert space $L^{2}(\Omega)$. We associate with problem (1.4) a densely defined, closed linear operator

$$
\mathfrak{A}: L^{2}(\Omega) \longrightarrow L^{2}(\Omega)
$$

as follows:

(1) $\mathcal{D}(\mathfrak{A})=\left\{u \in W^{2,2}(\Omega): B u=0\right.$ on $\left.\partial \Omega\right\}$.

(2) $\mathfrak{A} u=A u$ for every $u \in \mathcal{D}(\mathfrak{A})$.

Here and in the following the Sobolev space $W^{k, p}(\Omega)$ for $k \in \mathbf{N}$ and $1<p<$ $\infty$ is defined as follows:

$$
\begin{gathered}
W^{k, p}(\Omega)=\text { the space of functions } u \in L^{p}(\Omega) \text { whose derivatives } D^{\alpha} u, \\
|\alpha| \leq k, \text { in the sense of distributions are in } L^{p}(\Omega),
\end{gathered}
$$

and its norm $\|\cdot\|_{W^{k, p}(\Omega)}$ is given by the formula

$$
\|u\|_{W^{k, p}(\Omega)}=\left(\sum_{|\alpha| \leq k} \int_{\Omega}\left|D^{\alpha} u(x)\right|^{p} d x\right)^{1 / p} .
$$

Then we have the following fundamental spectral results (i), (ii) and (iii) of the operator $\mathfrak{A}$ (see [19, Theorem 5.1]):

(i) The operator $\mathfrak{A}$ is positive and selfadjoint in $L^{2}(\Omega)$.

(ii) Let $\lambda_{j}$ be the eigenvalues of the operator $\mathfrak{A}$ that are arranged in an increasing sequence

$$
\lambda_{1}<\lambda_{2} \leq \ldots \leq \lambda_{j} \leq \lambda_{j+1} \ldots,
$$

each eigenvalue being repeated according to its multiplicity. The first eigenvalue $\lambda_{1}$ is positive and algebraically simple, and its corresponding eigenfunction $\varphi_{1} \in C^{\infty}(\bar{\Omega})$ may be chosen to be strictly positive in $\Omega$. Namely, we have the assertions

$$
\begin{cases}A \varphi_{1}=\lambda_{1} \varphi_{1} & \text { in } \Omega \\ \varphi_{1}>0 & \text { in } \Omega, \\ B \varphi_{1}=0 & \text { on } \partial \Omega .\end{cases}
$$

(iii) No other eigenvalues $\lambda_{j}, j \geq 2$, have positive eigenfunctions.

To begin with, we state three well-known results about the homogeneous problem (1.3) under Dirichlet condition. We impose the following three conditions (P.1), (P.2) and (P.3) on the nonlinear term $p(\xi)$ :

(P.1) The function $p(\xi)$ is real-valued and of class $C^{2}$ on $\mathbf{R}$.

(P.2) $p(0)=0$.

(P.3) The limits $p^{\prime}( \pm \infty)=\lim _{\xi \rightarrow \pm \infty} p^{\prime}(\xi)$ exist.

If the eigenvalues $\mu_{j}$ of the operator $\mathfrak{A}$ in the Dirichlet case are arranged in an increasing sequence

$$
\mu_{1}<\mu_{2} \leq \ldots \leq \mu_{j} \leq \mu_{j+1} \ldots,
$$

then we have the following three results: 
(I) If there exist two positive constants $\gamma$ and $\gamma^{\prime}$ and a positive integer $n$ such that

$$
\mu_{n}<\gamma \leq p^{\prime}(\xi) \leq \gamma^{\prime}<\mu_{n+1} \quad \text { for all } \xi \in \mathbf{R},
$$

then the homogeneous problem (1.3) has only the trivial solution (Hammerstein [12, Satz 6]; Dolph [10, Theorem 3.1]; Castro-Lazer [7, Theorem D]).

(II) If there exists a positive integer $n$ such that

$$
\mu_{n-1}<p^{\prime}(0)<\mu_{n}<p^{\prime}( \pm \infty)<\mu_{n+1}
$$

and if $p(\xi)$ satisfies the condition

$$
\xi p^{\prime \prime}(\xi)>0 \text { for all } \xi \neq 0,
$$

then the homogeneous problem (1.3) has exactly two non-trivial solutions (Casto-Lazer [7, Theorem B]; Ambrosetti-Mancini [3, Theorem $1.2])$.

(III) Assume that $p^{\prime \prime}(\xi)>0$ on $\mathbf{R}$, that the limit $p^{\prime}(-\infty)=\lim _{\xi \rightarrow-\infty} p(\xi) / \xi$ exists and satisfies the condition

$$
0<p^{\prime}(-\infty)<\mu_{1}
$$

and further that the limit $p^{\prime}(+\infty)=\lim _{\xi \rightarrow+\infty} p(\xi) / \xi$ exists and satisfies the condition

$$
\mu_{1}<p^{\prime}(+\infty)<\mu_{2} .
$$

Then the homogeneous problem (1.3) has exactly one non-trivial solution if and only if $p^{\prime}(0) \neq \mu_{1}$ (Berger- Podolak [6, Theorem 3]).

We recall that the result (I) has been generalized to the degenerate case by Taira [24, Theorem 1.2]. The main purpose of this paper is to extend the results (II) and (III) to the degenerate case, by using the previous works Taira [20] through [24].

First, we impose the following three conditions (Q.1), (Q.2) and (Q.3) on the nonlinear term $p(\xi)$ :

(Q.1) The function $p(\xi)$ is real-valued and of class $C^{2}$ on $\mathbf{R}$, and $p(0)=0$.

(Q.2) $\xi p^{\prime \prime}(\xi)>0$ for all $\xi \neq 0$.

(Q.3) The limits $p^{\prime}( \pm \infty)=\lim _{\xi \rightarrow \pm \infty} p(\xi) / \xi$ exist and there exists a positive integer $n$ such that

$$
\lambda_{n-1}<p^{\prime}(0)<\lambda_{n}<p^{\prime}( \pm \infty)<\lambda_{n+1} .
$$

Example. A simple example of the nonlinear term $p(\xi)$ is given by the formula

$$
p(\xi)= \begin{cases}\frac{\lambda_{1}+\lambda_{2}}{2}\left(\xi+\frac{1}{2 \xi}-\frac{4}{3}\right) & \text { for } \xi>1, \\ \left(\frac{\lambda_{1}+\lambda_{2}}{12}\right) \xi^{3} & \text { for }-1 \leq \xi \leq 1, \\ \frac{\lambda_{1}+\lambda_{2}}{2}\left(\xi+\frac{1}{2 \xi}+\frac{4}{3}\right) & \text { for } \xi<-1 .\end{cases}
$$

It is easy to verify that this function $p(\xi)$ satisfies condition (1.5) for $n=1$ :

$$
p^{\prime}(0)=0<\lambda_{1}, \quad \lambda_{1}<p^{\prime}( \pm \infty)=\frac{\lambda_{1}+\lambda_{2}}{2}<\lambda_{2} .
$$


The next existence theorem is a generalization of the result (II) to the degenerate case:

Theorem 1.1. Assume that the nonlinear term $p(\xi)$ satisfies conditions (Q.1), (Q.2) and (Q.3). Then the homogeneous problem (1.3) has exactly three solutions, one trivial solution and exactly two non-trivial solutions $u_{1}, u_{2} \in$ $C^{2+\alpha}(\bar{\Omega})$ with exponent $0<\alpha<1$.

Rephrased, Theorem 1.1 asserts that the homogeneous problem (1.3) has exactly two non-trivial solutions provided that $p^{\prime}(\xi)$ crosses only the eigenvalue $\lambda_{n}$ of $\mathfrak{A}$ if $|\xi|$ goes from 0 to $\infty$.

Remark 1.2. Ambrosetti-Prodi [4] considered the case where the range of $p^{\prime}(\xi)$ contains only the first eigenvalue $\mu_{1}$ of the Dirichlet problem, and studied the non-homogeneous problem

$$
\begin{cases}A u-p(u)=h & \text { in } \Omega, \\ B u=0 & \text { on } \partial \Omega\end{cases}
$$

in the framework of singularity theory in Banach spaces ([17, Section 6]; [25]). They characterized completely the solution structure of problem (1.6) ([4, Theorem 3.1], [5, Chapter 4, Theorem 2.4]; see also [6, Theorem 3]). Their result is generalized to the degenerate case by Taira ([20, Theorem 1.1]).

Secondly, we consider the asymmetric case where the nonlinear term $p(\xi)$ has two different asymptotes as $\xi \rightarrow \pm \infty$ ([20]). More precisely, in addition to condition (Q.1), we impose the following three conditions (R.1), (R.2) and (R.3) on the nonlinear term $p(\xi)$ :

(R.1) $p^{\prime \prime}(\xi)>0$ on $\mathbf{R}$.

(R.2) The limit $p^{\prime}(-\infty)=\lim _{\xi \rightarrow-\infty} p(\xi) / \xi$ exists and satisfies the condition

$$
0<p^{\prime}(-\infty)<\lambda_{1}
$$

(R.3) The limit $p^{\prime}(+\infty)=\lim _{\xi \rightarrow+\infty} p(\xi) / \xi$ exists and satisfies the condition

$$
\lambda_{1}<p^{\prime}(+\infty)<\lambda_{2} \text {. }
$$

Example (K. Umezu). A simple example of the nonlinear term $p(\xi)$ is given by the formula

$$
p(\xi)=\left(\frac{\gamma^{\prime}+\gamma^{\prime \prime}}{2}\right) \xi+\sqrt{1+\frac{\left(\gamma^{\prime \prime}-\gamma^{\prime}\right)^{2}}{4} \xi^{2}}-1,
$$

where $\gamma^{\prime}$ and $\gamma^{\prime \prime}$ are positive constants such that

$$
0<\gamma^{\prime}<\lambda_{1}<\gamma^{\prime \prime}<\lambda_{2} .
$$

It is easy to verify that this function $p(\xi)$ satisfies the conditions

$$
p^{\prime}(-\infty)=\gamma^{\prime}, \quad p^{\prime}(+\infty)=\gamma^{\prime \prime}, \quad p^{\prime}(0)=\frac{\gamma^{\prime}+\gamma^{\prime \prime}}{2} .
$$

The next existence theorem is a generalization of the result (III) to the degenerate case: 
Theorem 1.3. In addition to condition (Q.1), we assume that the nonlinear term $p(\xi)$ satisfies conditions (R.1), (R.2) and (R.3). Then the homogeneous problem (1.3) has exactly two solutions, one trivial solution and exactly one non-trivial solution $u \in C^{2+\alpha}(\bar{\Omega})$ with exponent $0<\alpha<1$ if and only if the nonlinear term $p(\xi)$ satisfies the condition

$$
p^{\prime}(0) \neq \lambda_{1} .
$$

Rephrased, Theorem 1.3 asserts that the homogeneous problem (1.3) has exactly one non-trivial solution provided that $p^{\prime}(\xi)$ crosses only the first eigenvalue $\lambda_{1}$ of $\mathfrak{A}$ with $p^{\prime}(0) \neq \lambda_{1}$ if $\xi$ goes from $-\infty$ to $+\infty$.

The rest of this paper is organized as follows. In Section 2 we summarize the basic facts about the Leray-Schauder degree introduced by LeraySchauder [14] (Theorems 2.1 and 2.2)). In Section 3 we prove Theorem 1.1, by calculating explicitly the Leray-Schauder index (Lemma 3.6) and the LeraySchauder degree (Proposition 3.4) in Banach space, just as in AmbrosettiMancini [3]. The essential step in the proof is Lemma 3.5 which asserts that the Fréchet derivative $D \Phi$ of a nonlinear map $\Phi$ associated with the homogeneous problem (1.3) is invertible. It should be emphasized that our proof of Theorem 1.1 is based on the previous works [18], [20] and [21]. In particular, we make use of the comparison property of eigenvalues for degenerate elliptic boundary value problems with indefinite weights ([20, Corollary 3.6]). Section 4 is devoted to the proof of Theorem 1.3, which is inspired by BergerPodolak [6, Theorem 3]. We apply [20, Theorem 1.1] (see Theorem 4.1) to our situation.

The crucial point in our variational approach is to generalize the classical variational approach to eigenvalue problems with indefinite weights (cf. [9]) to the degenerate case, by using the theory of fractional powers of analytic semigroups. In fact, since the operator $\mathfrak{A}$ is positive and selfadjoint in the Hilbert space $L^{2}(\Omega)$, we can define its square $\operatorname{root} \mathcal{C}=\mathfrak{A}^{1 / 2}$ by the formula

$$
\mathcal{C} u=-\frac{1}{\pi} \int_{0}^{\infty} s^{-1 / 2}(s I+\mathfrak{A})^{-1} \mathfrak{A} u d s \quad \text { for all } u \in D(\mathfrak{A}),
$$

and introduce an underlying Hilbert space $\mathcal{H}$ as follows ([20, Theorem 3.1]):

$\mathcal{H}=$ the domain $D(\mathcal{C})$ with the inner product

$$
(u, v)_{\mathcal{H}}=(\mathcal{C} u, \mathcal{C} v)_{L^{2}(\Omega)} \quad \text { for all } u, v \in D(\mathcal{C})
$$

$=$ the completion of the domain $D(\mathfrak{A})$ with respect to the inner product

$$
\begin{array}{r}
(\mathfrak{A} u, v)_{L^{2}(\Omega)}=\sum_{i, j=1}^{N} \int_{\Omega} a^{i j}(x) \frac{\partial u}{\partial x_{i}} \frac{\partial v}{\partial x_{j}} d x+\int_{\Omega} c(x) u \cdot v d x \\
+\int_{\left\{a\left(x^{\prime}\right) \neq 0\right\}} \frac{b\left(x^{\prime}\right)}{a\left(x^{\prime}\right)} u \cdot v d \sigma \quad \text { for all } u, v \in D(\mathfrak{A}) .
\end{array}
$$

Here the last term on the right-hand side is an inner product of the Hilbert space $L^{2}(\partial \Omega)$ on the boundary $\partial \Omega$. 
The following diagram gives a bird's eye view of the right Hilbert space $\mathcal{H}$ for the variational approach (cf. [11]):

\begin{tabular}{|c|c|c|}
\hline$B$ & $\mathcal{H}$ & $a\left(x^{\prime}\right)$ and $b\left(x^{\prime}\right)$ \\
\hline The Dirichlet case & $W_{0}^{1,2}(\Omega)$ & $a\left(x^{\prime}\right) \equiv 0$ and $b\left(x^{\prime}\right) \equiv 1$ in $\Omega$ \\
\hline The Robin case & $W^{1,2}(\Omega)$ & $a\left(x^{\prime}\right) \equiv 1$ and $b\left(x^{\prime}\right) \not \equiv 0$ in $\Omega$ \\
\hline The degenerate case & $D\left(\mathfrak{A}^{1 / 2}\right)$ & (H.1) and (H.2) \\
\hline
\end{tabular}

\section{Topological Degree in Banach Space}

The Leray-Schauder degree is an important topological tool introduced by Leray and Schauder [14] in the study of nonlinear partial differential equations. The non-triviality of the degree guarantees the existence of a fixed point of the compact mapping in the domain. It should be emphasized that the more precisely we know the degree the sharper we can estimate the number of fixed points. This opens a door to the study of multiple solutions in nonlinear analysis (cf. [8], [15], [16], [17], [26]).

The Leray-Schauder degree is an extension of the Brouwer degree to mappings defined on an infinite dimensional real Banach space. The Brouwer fixed-point theorem asserts that a continuous map $f$ of a closed, bounded convex set $K \subset \mathbf{R}^{n}$ into itself has a fixed point. This is no longer true in infinite dimensions. In infinite-dimensional spaces, we must require more of $f$ than mere continuity.

Let $X$ be a real Banach space and let $\Omega$ be a bounded, open subset of $X$ with boundary $\partial \Omega$. A continuous map $f: \Omega \rightarrow X$ is said to be compact if it maps bounded sets in $\Omega$ into relatively compact sets of $X$. It should be emphasized that $f: \Omega \rightarrow X$ is compact if and only if $f$ is a uniform limit of mappings whose ranges lie in finite-dimensional subspaces.

The Leray-Schauder degree $\operatorname{deg}(f, \Omega, p)$ of a compact perturbation $f=$ $I-K$ of the identity map $I$ at a point $p \in X$ and relative to $\Omega$ can be defined by an analogue of the Galerkin approximation procedures, by assuming that

$$
f(x) \neq p \quad \text { on } \partial \Omega .
$$

Similar to the Brouwer degree, the Leray-Schauder degree enjoys the following properties:

(I) Normalization: If $I: X \rightarrow X$ is the identity map, then we have the assertion

$$
\operatorname{deg}(I, \Omega, p)= \begin{cases}1 & \text { if } p \in \Omega, \\ 0 & \text { if } p \notin \Omega .\end{cases}
$$

(II) Homotopy Invariance: Let $\Omega$ be a bounded open set in $X$. If $K: \bar{\Omega} \times$ $[0,1] \rightarrow X$ is compact and if a point $p \in X$ satisfies the condition

$$
x-K(x, t) \neq p \quad \text { for all } x \in \partial \Omega \text { and } 0 \leq t \leq 1,
$$

then it follows that $\operatorname{deg}(I-K(\cdot, t), \Omega, p)$ is independent of $t$. 
(III) Translation Invariance: For each point $p \in X$, we have the formula

$$
\operatorname{deg}(I-K, \Omega, p)=\operatorname{deg}(I-K-p, \Omega, 0) .
$$

(IV) Domain Additivity: If $\Omega_{1}$ and $\Omega_{2}$ are two open subsets in $\Omega$ such that $\Omega_{1} \cap \Omega_{2}=\emptyset$ and satisfy the condition

$$
x-K x \neq p \quad \text { for all } x \in \bar{\Omega} \backslash\left(\Omega_{1} \cup \Omega_{2}\right),
$$

then we have the formula

$$
\operatorname{deg}(I-K, \Omega, p)=\operatorname{deg}\left(I-K, \Omega_{1}, p\right)+\operatorname{deg}\left(I-K, \Omega_{2}, p\right) .
$$

The next theorem is a generalization of Kronecker's existence theorem for the Brouwer degree (see [8, Section 3.4]):

Theorem 2.1. Let $\Omega$ be a bounded open subset of a real Banach space $X$ and let $K: \bar{\Omega} \rightarrow X$ be compact. If $p_{0} \notin(I-K)(\partial \Omega)$ and $\operatorname{deg}\left(I-K, \Omega, p_{0}\right) \neq 0$, then there exists a point $x_{0} \in \Omega$ such that $(I-K) x_{0}=p_{0}$. In other words, if $p_{0} \in X$ satisfies the condition

$$
\operatorname{deg}\left(I-K, \Omega, p_{0}\right) \neq 0,
$$

then there exists a point $x_{0}$ in the closure $\bar{\Omega}$ such that $(I-K) x_{0}=p_{0}$.

Let $\phi$ be a mapping of the closure $\bar{\Omega}=\Omega \cup \partial \Omega$ into $X$ such that $\phi(x) \neq 0$ on $\partial \Omega$. Assume that

$$
\begin{aligned}
& \phi \in C^{1}(\Omega, X) \\
& K=I-\phi: \bar{\Omega} \rightarrow X \quad \text { is compact. }
\end{aligned}
$$

If $x_{0}$ is an isolated solution of the equation $\phi(x)=0$ and if the Fréchet derivative $D \phi\left(x_{0}\right)=I-D K\left(x_{0}\right)$ is invertible, we define the Leray-Schauder index of the map $\phi$ at $x_{0}, i\left(\phi, x_{0}, 0\right)$, by the formula

$$
i\left(\phi, x_{0}, 0\right)=\operatorname{deg}\left(\phi, B\left(x_{0}, \varepsilon\right), 0\right),
$$

where the open ball $B\left(x_{0}, \varepsilon\right)$ of radius $\varepsilon$ about $x_{0}$ is chosen so that it contains no other solution of the equation $\phi(x)=0$. It should be emphasized that the Leray-Schauder index $i\left(\phi, x_{0}, 0\right)$ does not depend on the special choice of $\varepsilon$.

The next index theorem plays an essential role in the proof of Theorem 1.1 (see [16, Theorem 2.8.1]):

Theorem 2.2 (the index theorem). Let $X$ be a real Banach space and let $\Omega$ be a bounded open set in $X$. If $\phi$ is a mapping of the closure $\bar{\Omega}=\Omega \cup \partial \Omega$ into $X$ such that $\phi(x) \neq 0$ on $\partial \Omega$ and if it satisfies the conditions

$$
\begin{aligned}
& \phi \in C^{1}(\Omega, X), \\
& K=I-\phi: \bar{\Omega} \longrightarrow X \quad \text { is compact, }
\end{aligned}
$$

then we have the formula for the Leray-Schauder index $i\left(\phi, x_{0}, 0\right)$

$$
i\left(\phi, x_{0}, 0\right)=(-1)^{\beta} \text {, }
$$

where

$$
\beta=\sum_{\mu_{j}>1} \beta_{j},
$$


$\beta_{j}$ being the algebraic multiplicity of the eigenvalue $\mu_{j}$ of the Fréchet derivative $D K\left(x_{0}\right)=I-D \phi\left(x_{0}\right)$.

\section{Proof of Theorem 1.1}

This section is devoted to the proof of Theorem 1.1. Our proof is inspired by Ambrosetti-Mancini [3, Theorem 1.2], and the crucial point in the proof is how to calculate the Leray-Schauder degree. The proof is based on the comparison property of eigenvalues for degenerate elliptic boundary value problems with indefinite weights ([20, Corollary 3.6]). The proof is divided into six steps.

Step I: We let

$$
X=C^{1}(\bar{\Omega})
$$

and transpose the homogeneous problem (1.3) into an equivalent nonlinear operator equation in $X$.

By using [18, Theorem 1.2] with $\varphi:=0$, we can define a linear operator (resolvent)

$$
L: X \longrightarrow X
$$

as follows: For each function $u \in X$, the function $v=L u \in C^{2+\alpha}(\bar{\Omega})$ is the unique solution of the boundary value problem

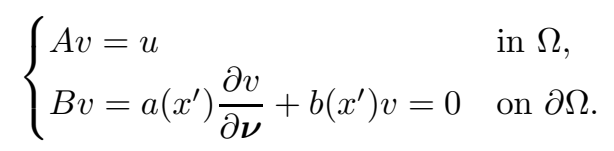

By the Ascoli-Arzelà theorem, it follows (see [19, Subsection 3.4]) that the resolvent $L: X \rightarrow X$ is compact.

Moreover, since the function $p(\xi)$ is of class $C^{2}$ on $\mathbf{R}$, we can introduce a nonlinear map

$$
P: X \longrightarrow X
$$

by the formula

$$
P(u)=p(u) \quad \text { for every } u \in X .
$$

The operator $P$ is called the Nemytskii operator associated with the nonlinear term $p(u)$.

Then it is easy to see that the homogeneous problem (1.3) is equivalent to the following operator equation:

$$
\Phi(u)=u-L(P(u))=0 \quad \text { in } X .
$$

Indeed, if a function $u \in X=C^{1}(\bar{\Omega})$ satisfies equation (3.1), then it follows from an application of $[18$, Theorem 1.2$]$ that

$$
u=L(P(u)) \in C^{2+\alpha}(\bar{\Omega}),
$$

since $P(u) \in C^{1}(\bar{\Omega})$.

First, the next lemma is an essential step in the proof of Theorem 1.1:

Proposition 3.1. Any solution $u$ of the equation $\Phi(u)=0$ is non-singular, that is, the Fréchet derivative $D \Phi(u)$ is invertible. 
Proof. If $u \in X$ is an arbitrary solution of $\Phi(u)=u-L(P(u))=0$, then it follows that $u \in C^{2+\alpha}(\bar{\Omega})$ and further that

$$
\begin{cases}A u=p(u) & \text { in } \Omega, \\ B u=0 & \text { on } \partial \Omega .\end{cases}
$$

It is easy to see that the Fréchet derivative $D \Phi(u)$ is given by the formula

$$
D \Phi(u) v=v-L\left(p^{\prime}(u) v\right) \quad \text { for every } v \in X .
$$

We remark that the Fréchet derivative $D \Phi(u)$ enjoys the Fredholm alternative, since $L: X \rightarrow X$ is compact and $p^{\prime}(\xi)$ is of class $C^{1}$ and bounded on R.

Therefore, we have only to show that $D \Phi(u)$ is injective. Namely, we have only to show that the linearized problem with the weight $p^{\prime}(u)$

$$
\begin{cases}A v=p^{\prime}(u) v & \text { in } \Omega, \\ B v=0 & \text { on } \partial \Omega\end{cases}
$$

has only the trivial solution.

Case (a): We consider the case where $u \neq 0$. First, we have, by conditions (Q.2) and (Q.3),

$$
p^{\prime}(\xi)<\lambda_{n+1} \quad \text { for all } \xi \in \mathbf{R},
$$

and so,

$$
p^{\prime}(u(x))<\lambda_{n+1} \quad \text { for all } x \in \bar{\Omega} .
$$

Hence it follows from an application of the comparison property of eigenvalues that

$$
\lambda_{n+1}\left(p^{\prime}(u)\right)>1 .
$$

If we introduce a function $\varphi(\xi)$ defined on $\mathbf{R}$ by the formula

$$
\varphi(\xi)= \begin{cases}\frac{p(\xi)}{\xi} & \text { if } \xi \neq 0, \\ p^{\prime}(0) & \text { if } \xi=0,\end{cases}
$$

then it follows from condition (Q.1) that $\varphi \in C^{1}(\mathbf{R})$. Moreover, we have, by problem (1.3),

This proves that

$$
\begin{cases}A u=\frac{p(u)}{u} \cdot u=\varphi(u) u & \text { in } \Omega, \\ B u=0 & \text { on } \partial \Omega .\end{cases}
$$

$$
\lambda_{j}(\varphi(u))=1 \text { for some } j \geq 1 .
$$

On the other hand, since we have, by conditions (Q.2) and (1.5),

$$
\lambda_{n-1}<\varphi(u(x))<\lambda_{n+1} \quad \text { for all } x \in \bar{\Omega},
$$

it follows from an application of the comparison property of eigenvalues that

$$
\lambda_{n-1}(\varphi(u))<1<\lambda_{n+1}(\varphi(u)) .
$$

Hence we have the assertion

$$
\lambda_{n}(\varphi(u))=1 .
$$


Moreover, we have, by conditions (Q.2) and (Q.3),

$$
\varphi(u(x))<p^{\prime}(u(x)) \text { for all } x \in \bar{\Omega},
$$

it follows from assertion (3.6) that

$$
\lambda_{n}\left(p^{\prime}(u)\right)<\lambda_{n}(\varphi(u))=1 .
$$

Therefore, by combining assertions (3.4) and (3.7) we obtain that

$$
\lambda_{n}\left(p^{\prime}(u)\right)<1<\lambda_{n+1}\left(p^{\prime}(u)\right),
$$

that is, 1 is not en eigenvalue of problem (3.3). This proves that problem (3.3) has only the trivial solution for $u \neq 0$.

Case (b): Secondly, we consider the case where $u=0$. It suffices to show that the linearized problem with the weight $p^{\prime}(0)$

$$
\begin{cases}A v=p^{\prime}(0) v & \text { in } \Omega \\ B v=0 & \text { on } \partial \Omega\end{cases}
$$

has only the trivial solution.

Since we have, by condition (1.5),

$$
\lambda_{n-1}<p^{\prime}(0)<\lambda_{n},
$$

it follows from an application of the comparison property of eigenvalues that

$$
\lambda_{n-1}\left(p^{\prime}(0)\right)<1<\lambda_{n}\left(p^{\prime}(0)\right) .
$$

Therefore, we obtain that 1 is not en eigenvalue of problem (3.9). This proves that problem (3.3) has only the trivial solution for $u=0$.

The proof of Proposition 3.1 is complete.

Step II: We calculate the Leray-Schauder index $i(\Phi, u, 0)$ :

Proposition 3.2. The Leray-Schauder index $i(\Phi, u, 0)$ is given by the formula

$$
i(\Phi, u, 0)= \begin{cases}(-1)^{n-1} & \text { if } u=0 \\ (-1)^{n} & \text { if } u \neq 0 .\end{cases}
$$

Proof. We apply the index theorem (Theorem 2.2) to the nonlinear map $\Phi$.

First, we consider the case where $u \neq 0$. In view of formula (3.2), we have only to study the eigenvalues $\lambda>1$ of the operator $L\left(p^{\prime}(u)\right)$.

It is easy to see that

$$
\begin{aligned}
L\left(p^{\prime}(u)\right) v=\lambda v & \Longleftrightarrow \begin{cases}\lambda A v=p^{\prime}(u) v & \text { in } \Omega, \\
B v=0 & \text { on } \partial \Omega\end{cases} \\
& \Longleftrightarrow \begin{cases}A v=\frac{1}{\lambda} p^{\prime}(u) v & \text { in } \Omega, \\
B v=0 & \text { on } \partial \Omega .\end{cases}
\end{aligned}
$$

Hence we have, for $j \geq 1$,

$$
\begin{aligned}
\frac{1}{\lambda}=\lambda_{j}\left(p^{\prime}(u)\right)<1 & \Longleftrightarrow \lambda=\frac{1}{\lambda_{j}\left(p^{\prime}(u)\right)}>1 \\
& \Longleftrightarrow \lambda_{j}\left(p^{\prime}(u)\right)<1 .
\end{aligned}
$$


However, it follows from assertion (3.8) that

$$
\lambda_{1}\left(p^{\prime}(u)\right)<\lambda_{2}\left(p^{\prime}(u)\right) \leq \ldots \leq \lambda_{n}\left(p^{\prime}(u)\right)<1<\lambda_{n+1}\left(p^{\prime}(u)\right),
$$

so that the number of eigenvalues $\lambda_{j}\left(p^{\prime}(u)\right)$ less than 1 is equal to $n$.

Therefore, by applying Theorem 2.2 to the map $\Phi$ we obtain that

$$
i(\Phi, u, 0)=(-1)^{n} \quad \text { if } u \neq 0 .
$$

Next, we consider the case where $u=0$. By assertion (3.10), it follows that

$$
\lambda_{1}\left(p^{\prime}(0)\right)<\lambda_{2}\left(p^{\prime}(0)\right) \leq \ldots \leq \lambda_{n-1}\left(p^{\prime}(0)\right)<1<\lambda_{n}\left(p^{\prime}(0)\right),
$$

so that the number of eigenvalues $\lambda_{j}\left(p^{\prime}(u)\right)$ less than 1 is equal to $n-1$. By Theorem 2.2, this proves that

$$
i(\Phi, u, 0)=(-1)^{n-1} \quad \text { if } u=0 .
$$

The proof of Proposition 3.2 is complete.

Step III: We prove that the set of solutions $u$ of the equation $\Phi(u)=0$ is bounded in the space $X=C^{1}(\bar{\Omega})$ :

Proposition 3.3. There exists a positive constant $R_{0}$ such that

$$
\Sigma=\{u \in X: \Phi(u)=0\} \subset B_{X}\left(0, R_{0}\right),
$$

where $B_{X}\left(0, R_{0}\right)=\left\{u \in X:\|u\|_{X}<R_{0}\right\}$ is the open ball of radius $R_{0}$ about 0 .

Proof. Assume, to the contrary, that there exists a sequence $\left\{u_{k}\right\}$ in $X$ such that $\Phi\left(u_{k}\right)=0$ and further that

$$
\left\|u_{k}\right\|_{X} \longrightarrow \infty \quad \text { as } k \rightarrow \infty .
$$

If we let

$$
z_{k}(x)=\frac{u_{k}(x)}{\left\|u_{k}\right\|_{X}} \quad \text { for every } x \in \bar{\Omega}
$$

then we obtain that

$$
\begin{cases}A z_{k}-\varphi\left(u_{k}\right) z_{k}=0 & \text { in } \Omega \\ B z_{k}=0 & \text { on } \partial \Omega\end{cases}
$$

where $\varphi(\xi)$ is the continuous function on $\mathbf{R}$ defined by formula (3.5).

Since we have, by conditions (Q.2) and (1.5),

$$
\lambda_{n-1}<\varphi\left(u_{k}(x)\right)<\lambda_{n+1} \quad \text { for all } x \in \bar{\Omega},
$$

and since $\left\|u_{k}\right\|_{X} \rightarrow \infty$ as $k \rightarrow \infty$, it follows that the functions

$$
\varphi\left(u_{k}(x)\right) z_{k}(x)
$$

are bounded in the space $C(\bar{\Omega})$. By applying [18, Theorem 1.1] for $p>N /(1-$ $\alpha$ ), we obtain from the equation

$$
z_{k}=L\left(\varphi\left(u_{k}\right) z_{k}\right) \quad \text { in } \Omega
$$


that the sequence $\left\{z_{k}\right\}$ is bounded in the Hölder space $C^{1+\alpha}(\bar{\Omega})$. Namely, we have, for some positive constant $C$,

$$
\left\|z_{k}\right\|_{C^{1+\alpha}(\bar{\Omega})} \leq C .
$$

Indeed, it follows from an application of Sobolev's imbedding theorem (see [1, Theorem 4.12, Part II]) that we have the imbedding

$$
W^{2, p}(\Omega) \subset C^{2-N / p}(\bar{\Omega}) \subset C^{1+\alpha}(\bar{\Omega}),
$$

for $p>N /(1-\alpha)$.

Therefore, by the Ascoli-Arzelà theorem we may assume that the sequence $\left\{z_{k}\right\}$ itself converges to some function $z^{*}$ in the space $C^{1}(\bar{\Omega})$ as $k \rightarrow \infty$ :

$$
z_{k} \longrightarrow z^{*} \text { in } C^{1}(\bar{\Omega}) \text { as } k \rightarrow \infty .
$$

We remark here that the limit function $z^{*}(x)$ satisfies the condition

$$
\left\|z^{*}\right\|_{X}=\lim _{n \rightarrow \infty}\left\|z_{k}\right\|_{X}=1
$$

and also the boundary condition

$$
B z^{*}=\lim _{n \rightarrow \infty} B z_{k}=0 \quad \text { on } \partial \Omega .
$$

On the other hand, by multiplying equation (3.12) by an arbitrary test function $w \in C_{0}^{\infty}(\Omega)$ and integrating over $\Omega$ we obtain that

$$
-\sum_{i, j=1}^{N} \int_{\Omega} a^{i j}(x) \frac{\partial z_{k}}{\partial x_{i}} \frac{\partial w}{\partial x_{j}} d x-\int_{\Omega} c(x) z_{k} w d x+\int_{\Omega} \varphi\left(u_{k}\right) z_{k} w d x=0 .
$$

However, we have, by condition (Q.3),

$$
\left\{\begin{array}{l}
(a) z^{*}(x)<0 \Longrightarrow u_{k}(x)=z_{k}(x)\left\|u_{k}\right\|_{X} \longrightarrow-\infty \quad \text { as } k \rightarrow \infty \\
\Longrightarrow \lim _{n \rightarrow \infty} \varphi\left(u_{k}(x)\right)=\lim _{n \rightarrow \infty} \frac{p\left(u_{k}(x)\right)}{u_{k}(x)}=p^{\prime}(-\infty) ; \\
(b) z^{*}(x)>0 \Longrightarrow u_{k}(x)=z_{k}(x)\left\|u_{k}\right\|_{X} \longrightarrow+\infty \quad \text { as } k \rightarrow \infty \\
\Longrightarrow \lim _{n \rightarrow \infty} \varphi\left(u_{k}(x)\right)=\lim _{n \rightarrow \infty} \frac{p\left(u_{k}(x)\right)}{u_{k}(x)}=p^{\prime}(+\infty) ; \\
(c) z^{*}(x)=0 \Longrightarrow \lim _{n \rightarrow \infty} z_{k}(x) \varphi\left(u_{k}(x)\right)=0 .
\end{array}\right.
$$

Hence, if we define a function $m(x)$ by the formula

$$
m(x)= \begin{cases}p^{\prime}(+\infty) & \text { if } z^{*}(x)>0 \\ \frac{\lambda_{n}+\lambda_{n+1}}{2} & \text { if } z^{*}(x)=0 \\ p^{\prime}(-\infty) & \text { if } z^{*}(x)<0\end{cases}
$$

then it follows that

$$
\varphi\left(u_{k}(x)\right) z_{k}(x) \longrightarrow m(x) z^{*}(x) \quad \text { in } \Omega \text { as } k \rightarrow \infty .
$$

Therefore, by applying the Lebesgue bounded convergence theorem we obtain from equation (3.15) that

$$
-\left(A z^{*}, w\right)_{L^{2}(\Omega)}+\left(m(x) z^{*}, w\right)_{L^{2}(\Omega)}
$$




$$
\begin{aligned}
& =-\sum_{i, j=1}^{N} \int_{\Omega} a^{i j}(x) \frac{\partial z^{*}}{\partial x_{i}} \frac{\partial w}{\partial x_{j}} d x-\int_{\Omega} c(x) z^{*} w d x+\int_{\Omega} m(x) z^{*} w d x \\
& =0 \quad \text { for all } w \in C_{0}^{\infty}(\Omega) .
\end{aligned}
$$

By combining three formulas (3.13), (3.14) and (3.18), we have proved that the non-trivial function $z^{*}(x)$ satisfies the conditions

$$
\begin{cases}A z^{*}=m(x) z^{*} & \text { in } \Omega, \\ B z^{*}=0 & \text { on } \partial \Omega .\end{cases}
$$

This proves that

$$
\lambda_{j}(m)=1 \quad \text { for some } j \geq 1 .
$$

However, since we have, by conditions (Q.2) and (1.5),

$$
\lambda_{n}<m(x)<\lambda_{n+1} \text { for almost all } x \in \Omega,
$$

it follows from an application of the comparison property of eigenvalues that

$$
\lambda_{n}(m)<1<\lambda_{n+1}(m) .
$$

This contradicts assertion (3.19).

Summing up, we have proved that the sequence $\left\{u_{k}\right\}$ in bounded in the space $X=C^{1}(\bar{\Omega})$.

The proof of Proposition 3.3 is complete.

Step IV: We calculate the Leray-Schauder degree $\operatorname{deg}\left(\Phi, B_{X}(0, R), 0\right)$ for some $R>R_{0}$ :

Proposition 3.4. There exists a positive constant $R>R_{0}$ such that the LeraySchauder degree $\operatorname{deg}\left(\Phi, B_{X}(0, R), 0\right)$ is given by the formula

$$
\operatorname{deg}\left(\Phi, B_{X}(0, R), 0\right)=(-1)^{n} .
$$

Proof. The proof of Proposition 3.4 is divided into four steps.

(1) We let

$$
m^{*}=\max \left\{p^{\prime}(+\infty), p^{\prime}(-\infty)\right\}
$$

and define a linear operator

$$
\widetilde{L}: X \longrightarrow X
$$

by the formula

$$
\widetilde{L} v=L\left(m^{*} v\right) \quad \text { for every } v \in X .
$$

Moreover, we introduce a one-parameter family $\{H(\cdot, t)\}_{0 \leq t \leq 1}$ of mappings as follows:

$$
H(u, t)=(1-t) \Phi(u)+t(u-\widetilde{L} u) \quad \text { for every } u \in X
$$

The next lemma is an essential step in the proof of Proposition 3.4:

Lemma 3.5. There exists a positive constant $R>R_{0}$ such that we have, for all $\|u\|_{X}=R$ and all $t \in[0,1]$,

$$
H(u, t) \neq 0 .
$$


Proof. Assume, to the contrary, that there exist a sequence $\left\{t_{k}\right\}$ in $[0,1]$ and a sequence $\left\{u_{k}\right\}$ in $X$ such that

$$
\begin{aligned}
& H\left(u_{k}, t_{k}\right)=\left(1-t_{k}\right) \Phi\left(u_{k}\right)+t_{k}\left(u_{k}-\widetilde{L} u_{k}\right)=0, \\
& \left\|u_{k}\right\|_{X} \longrightarrow+\infty \quad \text { as } k \rightarrow \infty
\end{aligned}
$$

If we let

then we obtain that

$$
z_{k}(x)=\frac{u_{k}(x)}{\left\|u_{k}\right\|_{X}} \quad \text { for every } x \in \bar{\Omega},
$$

$$
\left(1-t_{k}\right)\left(z_{k}-L\left(\varphi\left(u_{k}\right) z_{k}\right)\right)+t_{k}\left(z_{k}-\widetilde{L} z_{k}\right)=0,
$$

where $\varphi(\xi)$ is the continuous function on $\mathbf{R}$ defined by formula (3.5). Hence we have the formula

$$
z_{k}=\left(1-t_{k}\right) L\left(\varphi\left(u_{k}\right) z_{k}\right)+t_{k} \widetilde{L} z_{k}
$$

This implies that

$$
\begin{cases}A z_{k}=\left(1-t_{k}\right) \varphi\left(u_{k}\right) z_{k}+t_{k} m^{*} z_{k}, & \text { in } \Omega, \\ B z_{k}=0 & \text { on } \partial \Omega .\end{cases}
$$

Since $\varphi(\xi)$ is bounded on $\mathbf{R}$, it follows that the functions

$$
\left(1-t_{k}\right) \varphi\left(u_{k}(x)\right) z_{k}(x)+t_{k} m^{*} z_{k}(x)
$$

are bounded in the space $C(\bar{\Omega})$. Just as in the proof of Proposition 3.3, we obtain from the equation

$$
z_{k}=L\left(\left(1-t_{k}\right) \varphi\left(u_{k}\right) z_{k}+t_{k} m^{*} z_{k}\right) \quad \text { in } \Omega
$$

that the sequence $\left\{z_{k}\right\}$ is bounded in the Hölder space $C^{1+\alpha}(\bar{\Omega})$. Namely, we have, for some positive constant $C$,

$$
\left\|z_{k}\right\|_{C^{1+\alpha}(\bar{\Omega})} \leq C .
$$

Hence, by the Ascoli-Arzelà theorem we may assume that the sequence $\left\{z_{k}\right\}$ itself converges to some function $z^{*}$ in the space $C^{1}(\bar{\Omega})$ as $k \rightarrow \infty$, and further we may assume that the sequence $\left\{t_{k}\right\}$ itself converges to some point $t_{0}$ in the interval $[0,1]$ as $k \rightarrow \infty$ :

$$
\begin{aligned}
& z_{k} \longrightarrow z^{*} \quad \text { in } C^{1}(\bar{\Omega}) \text { as } k \rightarrow \infty, \\
& t_{k} \longrightarrow t_{0} \text { in }[0,1] \text { as } k \rightarrow \infty .
\end{aligned}
$$

Therefore, by passing to the limit in problem (3.22) we obtain from assertions (3.17), (3.23) and (3.24) that the limit function $z^{*}(x)$ satisfies the conditions

$$
\begin{cases}A z^{*}=\left(\left(1-t_{0}\right) m(x)+t_{0} m^{*}\right) z^{*}, & \text { in } \Omega, \\ B z^{*}=0 & \text { on } \partial \Omega, \\ z^{*}(x) \not \equiv 0 & \text { in } \Omega,\end{cases}
$$

where $m(x)$ is the function defined by formula (3.16). This proves that

$$
\lambda_{j}\left(\left(1-t_{0}\right) m(x)+t_{0} m^{*}\right)=1 \quad \text { for some } j \geq 1 \text {. }
$$


On the other hand, we have, by condition (1.5),

$$
\lambda_{n}<\left(1-t_{0}\right) m(x)+t_{0} m^{*}<\lambda_{n+1} \quad \text { for almost all } x \in \Omega .
$$

Hence it follows an application of the comparison property of eigenvalues that

$$
\begin{aligned}
& \lambda_{n}\left(\left(1-t_{0}\right) m(x)+t_{0} m^{*}\right)<1, \\
& \lambda_{n+1}\left(\left(1-t_{0}\right) m(x)+t_{0} m^{*}\right)>1,
\end{aligned}
$$

so that

$$
\lambda_{n}\left(\left(1-t_{0}\right) m(x)+t_{0} m^{*}\right)<1<\lambda_{n+1}\left(\left(1-t_{0}\right) m(x)+t_{0} m^{*}\right) .
$$

This contradicts formula (3.25).

The proof of Lemma 3.5 is complete.

(2) By virtue of Lemma 3.5, it follows from an application of the homotopy invariance of the degree that

$$
\begin{aligned}
\operatorname{deg}\left(\Phi, B_{X}(0, R), 0\right) & =\operatorname{deg}\left(H(\cdot, 0), B_{X}(0, R), 0\right) \\
& =\operatorname{deg}\left(H(\cdot, 1), B_{X}(0, R), 0\right) \\
& =\operatorname{deg}\left(I-\widetilde{L}, B_{X}(0, R), 0\right)
\end{aligned}
$$

(3) We calculate the degree $\operatorname{deg}\left(I-\widetilde{L}, B_{X}(0, R), 0\right)$ for $R>R_{0}$ :

Lemma 3.6. The degree $\operatorname{deg}\left(I-\widetilde{L}, B_{X}(0, R), 0\right)$ is given by the formula

$$
\operatorname{deg}\left(I-\widetilde{L}, B_{X}(0, R), 0\right)=(-1)^{n} .
$$

Proof. We apply the index theorem (Theorem 2.2) to the operator $I-\widetilde{L}$. To do this, we study the eigenvalues $\lambda>1$ of the operator $\widetilde{L}=m^{*} L$.

It is easy to see that

$$
\widetilde{L} v=\lambda v \Longleftrightarrow \begin{cases}A v=\frac{m^{*}}{\lambda} v & \text { in } \Omega, \\ B v=0 & \text { on } \partial \Omega .\end{cases}
$$

Hence we have, for $j \geq 1$,

$$
\lambda_{j}=\frac{m^{*}}{\lambda}, \lambda>1 \Longleftrightarrow \lambda=\frac{m^{*}}{\lambda_{j}}>1 \Longleftrightarrow \lambda_{j}<m^{*} .
$$

However, we obtain from formula (3.21) and assertion (3.26) with $t_{0}:=1$ that

$$
\lambda_{1}<\lambda_{2} \leq \ldots \leq \lambda_{n}<m^{*}<\lambda_{n+1},
$$

so that the number of eigenvalues $\lambda_{j}$ less than $m^{*}$ is equal to $n$. By Theorem 2.2 , this proves the desired formula (3.28).

(4) The desired formula (3.20) follows by combining formulas (3.27) and $(3.28)$.

The proof of Proposition 3.4 is complete. 
Step V: We prove that the set

$$
\Sigma=\{u \in X: \Phi(u)=0\}=\{u \in X: u-L(P(u))=0\}
$$

is a finite set. To do this, it suffices to show that $\Sigma$ is discrete and sequentially compact.

First, we find from Proposition 3.1 that the Fréchet derivative $D \Phi(u)=$ $I-L\left(p^{\prime}(u)\right)$ is invertible at every point $u$ of $\Sigma$. This implies that the set $\Sigma$ consists of isolated points, that is, it is discrete.

Furthermore, it is easy to see that $\Sigma$ is sequentially compact. Indeed, if $\left\{u_{n}\right\}$ is an arbitrary sequence in $\Sigma$, then it follows from an application of Proposition 3.3 that $\left\{u_{n}\right\}$ is bounded in $X$. However, since $P(\cdot): X \rightarrow X$ is continuous and since $L: X \rightarrow X$ is compact, we obtain that the sequence $\left\{L\left(P\left(u_{n}\right)\right\}\right.$ contains a convergent subsequence in $X$. This proves that the sequence $\left\{u_{n}\right\}=\left\{L\left(P\left(u_{n}\right)\right)\right\}$ contains a convergent subsequence in $X$.

Step VI: We prove that the homogeneous problem (1.3) has exactly three solutions.

Since the homogeneous problem (1.3) has one trivial solution, by Step $\mathrm{V}$ we may assume that

$$
\{u \in X: \Phi(u)=0\}=\left\{0, u_{1}, u_{2}, \ldots, u_{s}\right\} .
$$

Then, by using formula (3.11) (Lemma 3.6) and the domain additivity of the degree we find that

$$
\operatorname{deg}\left(\Phi, B_{X}(0, R), 0\right)=(-1)^{n-1}+s(-1)^{n}=(s-1)(-1)^{n} .
$$

Hence it follows from formula (3.20) (Proposition 3.4) that $s=2$, that is,

$$
\{u \in X: \Phi(u)=0\}=\left\{0, u_{1}, u_{2}\right\} .
$$

Summing up, we have proved that the homogeneous problem (1.3) has exactly three solutions, one trivial solution and exactly two non-trivial solutions $u_{1}, u_{2} \in C^{2+\alpha}(\bar{\Omega})$.

Now the proof of Theorem 1.1 is complete.

\section{Proof of Theorem 1.3}

This final section is devoted to the proof of Theorem 1.3. We remark that the homogeneous problem (1.3) has one trivial solution. The proof is divided into three steps.

Step I: We consider the semilinear non-homogeneous boundary value problem

$$
\begin{cases}A u-p(u)=h & \text { in } \Omega \\ B u=a\left(x^{\prime}\right) \frac{\partial u}{\partial \boldsymbol{\nu}}+b\left(x^{\prime}\right) u=0 & \text { on } \partial \Omega\end{cases}
$$

We let

$$
\begin{aligned}
X & =C_{B}^{2+\alpha}(\bar{\Omega})=\left\{u \in C^{2+\alpha}(\bar{\Omega}): B u=0 \quad \text { on } \partial \Omega\right\}, \\
Y & =C^{\alpha}(\bar{\Omega}),
\end{aligned}
$$


and introduce a nonlinear map

$$
F: X \longrightarrow Y
$$

by the formula

$$
F(u)=-A u+p(u) \quad \text { for every } u \in X .
$$

Then it is easy to see that problem (4.1) has a solution $u \in C^{2+\alpha}(\bar{\Omega})$ if and only if the operator equation

$$
F(u)=-h
$$

has a solution $u \in X$, and further that the homogeneous problem (1.3) is equivalent to the operator equation $F(u)=0$.

The essential step in the proof of Theorem 1.3 is the following existence theorem (see [20, Theorem 1.1]) which gives very exact results on the number of solutions of problem (4.1):

Theorem 4.1. Let the nonlinear term $p(\xi)$ be as in Theorem 1.3. Then there exist a $C^{1}$ manifold $Y_{1}$ of codimension one in $Y=C^{\alpha}(\bar{\Omega})$ and two disjoint open subsets $Y_{0}$ and $Y_{2}$ of $Y$ such that

$$
Y=Y_{0} \cup Y_{1} \cup Y_{2}
$$

with the following two properties:

(i) Problem (4.1) has a unique solution $u \in C^{2+\alpha}(\bar{\Omega})$ for any function $h \in Y_{1}$.

(ii) Problem (4.1) has exactly two solutions $u_{1}, u_{2} \in C^{2+\alpha}(\bar{\Omega})$ for any function $h \in Y_{2}$, while problem (4.1) has no solution for any function $h \in Y_{0}$.

It should be noticed (see [20, Theorem 2.3]) that the $C^{1}$ manifold $Y_{1}$ is given by the formula $Y_{1}=F\left(\Sigma^{\prime}\right)$ where

$\Sigma^{\prime}=\{u \in X$ : the Fréchet derivative $D F(u)$ is not invertible $\}$.

Step II: We prove that if the homogeneous problem (1.3) has exactly one non-trivial solution $u \in C^{2+\alpha}(\bar{\Omega})$, then $p^{\prime}(0) \neq \lambda_{1}$.

Since the homogeneous problem (1.3) has at least one trivial solution $u=0$, we have only to show that

$$
p^{\prime}(0)=\lambda_{1} \Longrightarrow h=0 \in Y_{1} \text {. }
$$

However, it is easy to verify that the Fréchet derivative $D F(0)$ at $u=0$ is given by the formula (cf. formula (3.2))

$$
D F(0) v=\left(-A+p^{\prime}(0)\right) v \quad \text { for every } v \in X .
$$

Hence, if $p^{\prime}(0)=\lambda_{1}$, we have the assertions

$$
\left\{\begin{array}{l}
0 \in \Sigma^{\prime}, \\
0=F(0) \in F\left(\Sigma^{\prime}\right)=Y_{1} .
\end{array}\right.
$$

This implies that $h=0 \in Y_{1}$.

Step III: We prove that

$$
h=0 \in Y_{1} \Longrightarrow p^{\prime}(0)=\lambda_{1} .
$$


This implies that if $p^{\prime}(0) \neq \lambda_{1}$, then the homogeneous problem (1.3) has one trivial solution and exactly one non-trivial solution $u \in C^{2+\alpha}(\bar{\Omega})$.

To do this, we make use of the following orthogonal decomposition of $Y$ (see $[21$, formula $(3.2)])$ :

$$
\begin{aligned}
Y & =C^{\alpha}(\bar{\Omega}) \\
& =\left\{u \in X:\left(A-\lambda_{1}\right) u=0 \text { in } \Omega\right\} \oplus\left\{\left(A-\lambda_{1}\right) u: u \in X\right\} \\
& :=\operatorname{span}\left[\phi_{1}\right] \oplus W .
\end{aligned}
$$

Here we recall that $\phi_{1}$ is the eigenfunction of the operator $\mathfrak{A}$ in $C^{2+\alpha}(\bar{\Omega})$ such that

$$
\begin{cases}A \phi_{1}=\lambda_{1} \phi_{1} & \text { in } \Omega \\ \phi_{1}>0 & \text { in } \Omega \\ B \phi_{1}=0 & \text { on } \partial \Omega\end{cases}
$$

and that

$$
W=\left\{\left(A-\lambda_{1}\right) u: u \in X\right\} .
$$

If we define the orthogonal projection $Q$ from $X$ onto $W$ by the formula

$$
Q u=u-\left(\int_{\Omega} u(x) \phi_{1}(x) d x\right) \phi_{1} \quad \text { for every } u \in X,
$$

then it is easy to see that

$$
W=Q(X)=\left\{w \in Y: \int_{\Omega} w(x) \phi_{1}(x) d x=0\right\} .
$$

Moreover, by restricting decomposition (4.2) to the subspace $X$ of $Y$ we obtain the orthogonal decomposition

$$
X=C_{B}^{2+\alpha}(\bar{\Omega})=\operatorname{span}\left[\phi_{1}\right] \oplus(W \cap X) .
$$

In other words, every function $u \in X$ can be written uniquely in the form

$$
u=t \phi_{1}+w(t), \quad t=\int_{\Omega} u(x) \phi_{1}(x) d x, \quad w(t) \in X \cap W .
$$

Now we assume that $h \in Y_{1}$. Then it follows that a unique solution $u$ of the homogeneous problem (1.3) can be expressed as follows:

$$
u=t \phi_{1}+w(t, h), \quad w(t, h) \in X \cap W,
$$

where the function $w(t, h)$ satisfies the equation

$$
A\left(t \phi_{1}+w(t, h)\right)-g\left(t \phi_{1}+w(t, h)\right)=h .
$$

Moreover, we remark that the function $w(t, h)$ satisfies the condition

$$
w(0,0)=0,
$$

since $h=0 \in Y_{1}$. By differentiating both sides of equation (4.3) at $t=0$ and taking $h=0$, we obtain from condition (4.4) that

$$
\begin{aligned}
0 & =A\left(\phi_{1}+w_{t}^{\prime}(0,0)\right)-p^{\prime}(0)\left(\phi_{1}+w_{t}^{\prime}(0,0)\right) \\
& =\left(\lambda_{1}-p^{\prime}(0)\right) \phi_{1}+A w_{t}^{\prime}(0,0)-p^{\prime}(0) w_{t}^{\prime}(0,0),
\end{aligned}
$$


with

$$
\left(\lambda_{1}-p^{\prime}(0)\right) \phi_{1} \in \operatorname{span}\left[\phi_{1}\right], \quad A w_{t}^{\prime}(0,0)-p^{\prime}(0) w_{t}^{\prime}(0,0) \in W .
$$

By decomposition (4.2), this implies that

$$
\left\{\begin{array}{l}
\left(\lambda_{1}-p^{\prime}(0)\right) \phi_{1}=0 \\
A w_{t}^{\prime}(0,0)-p^{\prime}(0) w_{t}^{\prime}(0,0)=0
\end{array}\right.
$$

so that

$$
\lambda_{1}=p^{\prime}(0), \quad w_{t}^{\prime}(0,0)=0 .
$$

Summing up, we have proved that the homogeneous problem (1.3) has exactly one non-trivial solution $u \in C^{2+\alpha}(\bar{\Omega})$ if and only if the nonlinear term $p(\xi)$ satisfies condition (1.7).

The proof of Theorem 1.3 is complete.

\section{References}

[1] R.A. Adams and J.J.F. Fournier, Sobolev spaces, second edition, Academic Press, Amsterdam Heidelberg New York Oxford, 2003.

[2] H. Amann, Fixed point equations and nonlinear eigenvalue problems in ordered Banach spaces, SIAM Rev. 18 (1976) 620-709.

[3] A. Ambrosetti and G. Mancini, Sharp nonuniqueness results for some nonlinear problems, Nonlinear Anal. 3 (1979) 635-645.

[4] A. Ambrosetti and G. Prodi, On the inversion of some differentiable mappings with singularities between Banach spaces, Ann. Mat. Pura Appl. 93 (1972) 231-247.

[5] A. Ambrosetti and G. Prodi, A primer of nonlinear analysis, Cambridge Stud. Adv. Math. Cambridge University Press, Cambridge, 1993.

[6] M.S. Berger and E. Podolak, On the solutions of a nonlinear Dirichlet problem, Indiana Univ. Math. J. 24 (1975) 837-846.

[7] A. Castro and A. C. Lazer, Critical point theory and the number of solutions of a nonlinear Dirichlet problem, Ann. Mat. Pura Appl. 120 (1979) 113-137.

[8] K.C. Chang, Methods in nonlinear analysis, Springer Monogr. Math. SpringerVerlag, Berlin Heidelberg New York, 2005.

[9] D.G. de Figueiredo, Positive solutions of semilinear elliptic problems, In: Differential equations, Lecture Notes in Math. No. 957, 34-87, Springer-Verlag, Berlin Heidelberg New York, 1982.

[10] C.L. Dolph, Nonlinear integral equations of the Hammerstein-type, Trans. Amer. Math. Soc. 66 (1949) 289-307.

[11] D. Fujiwara, Concrete characterization of the domains of fractional powers of some elliptic differential operators of the second order, Proc. Japan Acad. Ser. A Math. Sci. 43 (1967) 82-86.

[12] A. Hammerstein, Nichtlineare Integralgleichungen nebst Anwendungen, Acta Math. 54 (1930) 117-176.

[13] L. Hörmander, The analysis of linear partial differential operators III, Grundlehren Math. Wiss. Springer-Verlag, Berlin Heidelberg New York Tokyo, 1985. 
[14] J. Leray and J. Schauder, Topologie et équations fonctionelles, Ann. Sci. École Norm. Sup. 51 (1934) 45-78.

[15] J. Mawhin, Topological degree methods in nonlinear boundary value problems, CBMS Reg. Conf. Ser. in Math. no. 40, American Mathematical Society, Providence, Rhode Island, 1979.

[16] L. Nirenberg, Topics in nonlinear functional analysis, Courant Institute of Mathematical Sciences, New York University, New York, 1974.

[17] T. Runst and W. Sickel, Sobolev spaces of fractional order, Nemytskij operators, and nonlinear partial differential equations, Walter de Gruyter, Berlin New York, 1996.

[18] K. Taira, Semigroups, boundary value problems and Markov processes, Springer Monogr. Math. Springer-Verlag, Berlin Heidelberg New York, 2004.

[19] K. Taira, Degenerate elliptic boundary value problems with indefinite weights, Mediterr. J. Math. 5 (2008) 133-162.

[20] K. Taira, Degenerate elliptic boundary value problems with asymmetric nonlinearity, J. Math. Soc. Japan 62 (2010) 431-465.

[21] K. Taira, Semilinear degenerate elliptic boundary value problems at resonance, Ann. Univ. Ferrara Sez. VII Sci. Mat. 56 (2010) 369-392.

[22] K. Taira, Multiple solutions of semilinear degenerate elliptic boundary value problems, Math. Nachr. 284 (2011) 105-123.

[23] K. Taira, Multiple solutions of semilinear degenerate elliptic boundary value problems II, Math. Nachr. 284 (2011) 1554-1566.

[24] K. Taira, Degenerate elliptic boundary value problems with asymptotically linear nonlinearity, Rend. Circ. Mat. Palermo 60 (2011) 283-308.

[25] R. Thom, Les singularités des applications différentiables, Ann. Inst. Fourier (Grenoble) 6 (1955-1956) 43-87.

[26] E. Zeidler, Nonlinear functional analysis and its applications I, Springer-Verlag, New York Berlin Heidelberg, 1986.

\section{Acknowledgment}

The author is grateful to the referee for many valuable suggestions which improved the presentation of this paper. This research was partially supported by Grant-in-Aid for General Scientific Research (No. 19540162), Ministry of Education, Culture, Sports, Science and Technology, Japan.

Kazuaki Taira

Institute of Mathematics

University of Tsukuba

Tennodai 1-1-1

Tsukuba 305-8571

Japan

e-mail: taira@math.tsukuba.ac.jp 